\title{
Clima social familiar y rendimiento académico, en la institución educativa José Félix Black de Paiján
}

\author{
Family social climate and academic performance, in the educational institution \\ José Félix Black de Paiján
}

Delsi Victoria Velásquez Amaya delcy

delsi_victoria@hotmail.com

Código ORCID: 0000-0003-1594-8139

Universidad Peruana Unión, Perú
Ana Rebeca Escobedo Ríos

anaescobedo@upeu.edu.pe

Código ORCID: 0000-0002-6075-3325

Universidad Peruana Unión, Perú

Recibido: octubre 2020 / Arbitrado: noviembre 2020 / Publicado: enero 2021

RESUMEN

El objetivo de este estudio fue determinar la relación del Clima social familiar y el rendimiento académico de los estudiantes de la Institución Educativa José Félix Black de Paiján, La Libertad. La metodología correspondió al enfoque cuantitativo con un diseño trasversal y correlacional en 260 alumnos con el test de Moos. Los resultados expresaron que sólo el 37,7\% tienen un rendimiento regular, el $41,5 \%$ en un nivel bueno y $18,8 \%$ en niveles bajo. En conclusión el presente estudio corroboró el resultado de otros estudios (Velásquez 2014; Gonzales, 2006, Coello 2010). Aunque la variable rendimiento académico es un constructo sensible a otros factores; el clima social familiar impacta en algún modo en que el alumno tenga mejor eficiencia en sus estudios, además de considerar que el desarrollo de un buen clima familiar esté supeditado a factores culturales de la zona, además de la educación, religión y otros que lo configuran.

Palabras clave: Clima familiar; desarrollo familiar; relaciones familiares; estabilidad familiar rendimiento académico

The objective of this study was to determine the relationship between the family climate and the academic performance of the students of the José Félix Black Educational Institution in Paiján, La Libertad. The methodology corresponded to the quantitative approach with a transversal and correlational design in 260 students with the Moos test. The results expressed that only $37.7 \%$ have a regular performance, $41.5 \%$ at a good level and $18.8 \%$ at a low level. In conclusion, the present study corroborated the result of other studies (Velásquez 2014; Gonzales, 2006, Coello 2010). Although the academic performance variable is a sensitive construct to other factors; The family social climate impacts in some way in which the student has better efficiency in their studies, in addition to considering that the development of a good family climate is subject to cultural factors of the area, in addition to education, religion and others that configure it .

Youth violence; youth depression; hostility; anger; verbal aggression; physical aggression 


\section{INTRODUCCIÓN}

En América Latina a pesar de la existencia de importantes diferencias entre países es posible sostener que el rendimiento de los estudiantes latinoamericanos es bajo en Lengua como en Matemática, tanto en primaria como en secundaria. En países donde se ha evaluado el rendimiento a través de pruebas criteriales, se refleja un elevado porcentaje de alumnos que se ubican por debajo del Nivel de desempeño establecido como suficiente $\mathrm{o}$, dicho de otro modo, se expresa en el bajo porcentaje de estudiantes que logran los aprendizajes esperados de acuerdo a su grado y escolaridad previa. En efecto, los resultados de los países que han definido un nivel de suficiencia mínimo a alcanzar por los estudiantes en las áreas evaluadas, demuestran, inequívocamente, dicha afirmación (Murillo, 2008).

Asimismo, en Honduras en $6^{\circ}$ grado, alcanzan el nivel definido como suficiente un $11,1 \%$ de los alumnos en Lengua (español) y un $7,6 \%$ en Matemática. En Guatemala, por último el $55 \%$ y del $47 \%$ de los alumnos que alcanza el nivel de suficiencia predeterminado en Matemática y Lengua respectivamente. En Perú, por ejemplo, sólo el 7,9\% de los alumnos de $6^{\circ}$ de Primaria y el $6,0 \%$ de los que cursan el $3^{\circ}$ de secundaria logran los aprendizajes esperados para su nivel en Matemática (Murillo, 2008).

También es necesario resaltar que en nuestro país el $30 \%$ de la población estudiantil de la zona rural muestran atraso escolar y en estudiantes con lenguas nativas el porcentaje de atraso escolar es mayor alcanzando un promedio de $49 \%$.En nuestra región a pesar de que en los últimos años se ha logrado un avance significativo en el rendimiento académico según las estadísticas todavía existe un $18 \%$ de retraso escolar (Misterio de Educación, 2008).

El rendimiento académico refleja el grado de aprendizaje que el alumno adquiere ante una determinada situación de exigencia académica dentro de un proceso educativo y un periodo de tiempo establecido por los agentes inmediatos de la educación básica regular. Sin embargo, los registros y actas de evaluación muestran que el rendimiento académico de los estudiantes es un resultado insatisfactorio para los docentes, padres de familia y la Institución Educativa.

Se han considerado diversos aspectos sociales y educativos como posibles determinantes del rendimiento académico, entre ellos el clima social familiar (Mizala, 2002).

Al interior de la familia se estructuran un conjunto de demandas funcionales a sus miembros y una exigencia a los hijos del cumplimiento de su rol de estudiantes; por lo tanto el rendimiento académico, es una de las demandas alrededor del cual se configuran los niveles de interacción $y$, si el estudiante alcanza un rendimiento escolar óptimo, evidencia fracasos 0 rinde pobremente en la escuela, mucho tiene que ver en este aspecto el clima familiar dentro del cual se desenvuelven los hijos.

Es decir, la familia tiene una relación directa con diversas funciones del desarrollo humano entre ellas el aprendizaje y su expresión de avance como lo constituye el rendimiento. Musitu, Román y Gracia (1988, citado en López- Justicia, Pichardo; Fernández de Haro y J.A. Amezcua, 2000) corroboran 
que: "La familia representa para el individuo un sistema de participación y exigencias; un contexto, donde se generan y expresan emociones; un medio donde se proporcionan satisfacciones y se desempeñan funciones relacionadas con la educación y cuidado de los hijos"

\section{Antecedentes}

Coello (2010) estudió la incidencia de la estructura y clima familiar en el rendimiento escolar de los alumnos de octavo, noveno y décimo año de la Unidad Educativa Salesiana José Félix Pintado en Macas-Ecuador en el que se observa que el "66\% de alumnos que provienen de hogares completos aprobaron el año sin complicaciones y en el $34 \%$ de estudiantes que provienen de hogares desintegrados están el $18 \%$ de no promovidos $y$ el $16 \%$ que fueron promovidos con dificultad".

Por su parte, Fuentes (2003, citado por Gonzales y Pereda, 2006) realizó un estudio con el objetivo de determinar la influencia del Clima Afectivo Familiar en el Rendimiento Escolar de los alumnos del $3^{\circ}$ y $7^{\circ}$ años de la Escuela "Emilia Romagna de Traiguén", para el cual aplicó un diseño no experimental de corte transversal, correlacional, arribando a la conclusión que; "El clima Afectivo Familiar así como la falta de preocupación de los padres por los deberes escolares de sus hijos(as) influye en el rendimiento escolar". Los resultados permiten comprobar que: "en las familias de estos alumnos, se vivencia un clima negativo, por cuanto experimentan una carencia de afecto, cariño y ternura, padres centrados más en lo negativo y la crítica, familias que no ofrecen seguridad y confianza a sus hijos" (s.n.)

\section{El clima social familiar}

El clima social familiar es una de las más importantes conceptualizaciones en el estudio de la conducta humana en contextos sociales. Surgido de la Ecología Social, se constituyó en un enfoque que estudia la conducta humana desde diferentes perspectivas. El clima socio familiar de los estudiantes se ve condicionado por muchos factores, que determinan los comportamientos de los integrantes de la familia, toda vez que ellos adoptan conductas inadecuadas, la familia sufre crisis socio emocionales y rompen toda relación amical que significa frustrar los objetivos propuestos en la familia.

Para ampliar lo antes mencionado es pertinente destacar que Litvin (1966); Meyer (1968) y Schneider (1968) formularon para su análisis, algunas dimensiones de clima y su estrecha relación con la estimulación de los trabajadores. Williams (1993) señala lo siguiente:

Los resultados confirmaron que las dimensiones, tales como la estructura, responsabilidad, calidez humana y el apoyo, reconocimiento y recompensa, tolerancia para los conflictos, identidad y lealtad hacia la organización y el riesgo, constituían las dimensiones que pueden reducir, aumentar o sencillamente no afectar a las motivaciones hacia el logro, a la afiliación y al poder de las personas en una organización. (p.24) 
El clima social ha sido también estudiado por Moos (1975, 1976, 1979, 1981). El autor ha desarrollado 180 investigaciones realizadas en ambientes hospitalarios, residencias estudiantiles, comunidades terapéuticas, centros de rehabilitación, centros psiquiátricos y prisiones norteamericanas, con el fin de analizar las modificaciones de conducta que se producen en las personas como resultado de los cambios en algunas dimensiones importantes en el clima social en el cual se desarrollan programas de tratamiento.

También señala que una específica familia puede ser más o menos controladora, cohesiva, organizada, etc. El objetivo de Moos $y$ sus colegas ha sido encontrar invariantes detalles a través de diferentes ambientes sociales. (Fernández, 1998, p. 144).

\begin{abstract}
Al evaluar a los sujetos en contextos específicos podemos identificar y caracterizar la gama de relaciones interpersonales percibidas y valoradas por aquellos. Uno de los aspectos más importantes al abordar el estudio del clima, es la posibilidad desvincularlo con otras variables, ya no desde la perspectiva de lo percibido, sino centradas en características objetivas. (Cassullo, Álvarez y Pasman, 1998, p.187)
\end{abstract}

Más adelante, Moos ha desarrollado una serie de trabajos que han permitido establecer las más importantes dimensiones del clima social. Según Fernández (1998) Los estudios sobre clima social fueron realizados en diez diferentes ambientes sociales:1) Correccionales de adultos y 2 ) adolescentes,
3) Compañías militares,4) Residencias de ancianos, 5) Contextos educativos, 6) Colegios mayores universitarios, 7) Escuelas, 8) Ambientes comunitarios, 9) Familia, 10) Trabajo.

De los estudios anteriores se desarrolló la escala del clima social escolar, la cual fue el instrumento de esta investigación. Respecto a la Escala de clima social familiar (Family Environment Scale FES), muchos autores coinciden en afirmar que la interacción de los miembros de la familia produce algo que ha dado en llamarse clima. Este término ha sido trabajado con mayor profundidad por Moos, Freedman y Bronfrembrenner, cuyos estudios han caracterizado algunos elementos del funcionamiento de las familias y han demostrado que existe relación entre el clima social y la conducta de los miembros.

El componente básico del microsistema familiar, la díada, hace posible la formación de estructuras más grandes y por otro lado constituye un contexto crítico para el desarrollo por sí misma. Posteriormente para Bronfrembrenner, se establece una relación cuando una persona en un entorno presta atención a las actividades de otro o participa en ellas. La presencia de una relación en ambas direcciones cumple la condición mínima y definitiva para la existencia de una díada.

Bronfrembrenner (1976) ha realizado extensos estudios para demostrar desde un enfoque eco sistémico, cómo el proceso intrafamiliar se ve fuertemente influido por el ambiente externo. Por su parte, Williams (1993) en Venezuela destaca la importancia de la interrelación intrafamiliar y resalta lo siguiente: 
la familia es más que un mero conjunto de personas que se relacionan, e interactúan con sus propios objetivos, motivaciones, concepciones de su propia individualidad y de la de los demás, también enfatiza que cada miembro de la familia difiere en la habilidad para auto dirigirse, simbolizar, pensar $y$ percibir su mundo familiar.

La teoría del Clima Social de Moos refiere Kemper (2000), tiene como fundamento a la teoría del Clima Social de Rudolf Moos (1984) y ésta tiene como base teórica a la psicología ambientalista.

\section{Dimensiones y Áreas del clima Social Familiar.}

Kemper (2000) sostiene que el clima social que es un concepto y cuya operacionalización resulta difícil de universalizar, pretende describir las características psicológicas e institucionales de un determinado grupo humano situado sobre un ambiente. Citando a Moos, R. (1984), manifiesta que para estudiar o evaluar el clima social familiar, son tres las dimensiones o atributos afectivos que hay que tener en cuenta, para lo cual ha elaborado diversas escalas de Clima Social aplicables a diferentes tipos de ambiente, como es el caso de la Escala del Clima Social en la Familia (FES). En ella se trata sobre las interrelaciones que se dan entre los miembros de la familia donde se cumplen las funciones de comunicación, interacción, etc. El desarrollo personal puede ser fomentado por la vida en común, así como la organización y el grado de control que se ejercen unos miembros sobre otros. Cuyas características son:

- Relaciones: es la dimensión que evalúa el grado de comunicación y libre expresión dentro de la familia y el grado de interacción conflictiva que la caracteriza. Está integrado por 3 subescalas: cohesión, expresividad y conflicto: (i) Cohesión; mide el grado en el que los miembros del grupo familiar están compenetrados y se apoyan entre sí. (ii) Expresividad; explora el grado en el que se permite y anima a los miembros de la familia a actuar libremente y a expresar directamente sus sentimientos. (iii) Conflicto; grado en el que se expresan libre y abiertamente la cólera agresividad y conflicto entre los miembros de la familia.

- Desarrollo: evalúa la importancia que tienen dentro de la familia ciertos procesos de desarrollo personal, que pueden ser permitidos o no por la vida en común. Esta dimensión comprende las sub escalas de autonomía, actuación, intelectual-cultural y moralidad-religiosidad: (i) Autonomía; grado en el que los miembros de la familia están seguros de sí mismos, son independientes y toman sus propias decisiones. (ii) Actuación; grado en el que las actividades, tales como el colegio o el trabajo se enmarcan en una estructura orientada a la acción. (iii) Competencia. Intelectual - Cultural; grado de interés en las en las actividades de tipo político, intelectual y cultural y social. (iiii) 
Moralidad - Religiosidad; importancia que se le da a las prácticas y valores de tipo ético y religioso.

- Estabilidad: proporciona informaciones sobre la estructura y organización de la familia y sobre el grado de control que normalmente ejercen unos miembros de la familia sobre otros. Lo forman dos sub-escalas: organización y control: (i) Organización; importancia que se le da en el hogar a una clara, organización y estructura al planificar las actividades y responsabilidades de la familia. (ii) Control; grado en el que la dirección de la vida familiar se atiene a reglas $y$ procedimientos establecidos.

\section{MÉTODO}

El presente estudio se realizó bajo un enfoque cuantitativo de tipo no experimental de diseño correlacional y transversal. Para determinar el tamaño de la muestra se seleccionara en forma aleatoria $y$ probabilística simple considerando el tamaño de la población y se realizará un cálculo a través del algoritmo para poblaciones finitas siguiente: Una vez calculado el tamaño de la muestra 218 y ampliado a 260 se procederá a distribuirlo proporcionalmente en cada una de las 16 secciones de los cinco grados del nivel secundario. Para tal efecto, se requiere establecer la frecuencia del muestreo para determinar el tamaño de cada estrato, a través de la fórmula.

Con respecto a la recolección de la información se llevó a cabo a través de la técnica de la encuesta y el cuestionario restringido o cerrado como instrumental de investigación científica. El cuestionario consistirá en el uso dos instrumentos estandarizados tales como: La Escala de Clima Social Familiar (Family Environment Scale Fes) de Moos, A continuación se describe los instrumentos a utilizar:

La Escala de Clima Social Familiar(Family Enviroment Scale Fes de Moos); de la misma manera para el rendimiento académico se usó los registro de notas del colegio en estudio.

\section{Confiabilidad del instrumento}

Se utilizó para la recolección de datos el test de Moos descrito líneas antes un cuestionario muy utilizado en el Perú estandarizado en estudios similares; sin embargo, se procedió a su revisión para una validación en este contexto, validándose por juicio de expertos 03 profesionales especializados al respecto, (un metodólogo, un educador y un estadista) luego se realizó la prueba piloto y seguidamente la confiabilidad estadística nos arrojó una confiabilidad mediante alfa de Cronbach de 0,605.

Los datos recolectados se procesaron mediante el paquete estadístico computacional Statistical Package of the Social Sciences (SPSS), versión 22. Las técnicas estadísticas utilizadas para el análisis de los datos y consolidar los objetivos del estudio se hicieron mediante análisis descriptivos e inferenciales: Tablas de frecuencias para el aspecto descriptivo; tablas cruzadas y prueba Chi Cuadrado $\left(X^{2}\right)$ para la asociación. Para la verificación de la relación entre variables, se utilizó el coeficiente de correlación de Rho Spearman. Los resultados se interpretaron de acuerdo con los objetivos del estudio y las hipótesis, los mismos que son expuestos mediante tablas y figura. 
RESULTADOS

Los hallazgos del proceso investigativo se presentan a continuación en Tablas con su respectiva interpretación.

Tabla 1. Clima social familiar

\begin{tabular}{lcccc}
\hline & Frecuencia & Porcentaje & $\begin{array}{c}\text { Porcentaje } \\
\text { válido }\end{array}$ & $\begin{array}{c}\text { Porcentaje } \\
\text { acumulado }\end{array}$ \\
\hline Significativamente & 1 &, 4 &, 4 &, 4 \\
bajo & 10 & 3,8 & 3,8 & 4,2 \\
Bajo & 216 & 83,1 & 83,1 & 87,3 \\
Promedio & 31 & 11,9 & 11,9 & 99,2 \\
Alto & 2 &, 8 &, 8 & 100,0 \\
Significativamente alto & 260 & 100,0 & 100,0 & \\
Total & & & &
\end{tabular}

En la Tabla 1 se observa que en el clima social familiar de los estudiantes, se da en nivel promedio en un $83,1 \%$; los niveles altos de clima social familiar solo se representan en un $11,9 \%$ y tan sólo 3,8\% en niveles bajos.

Tabla 2. Rendimiento académico

\begin{tabular}{lcccc}
\hline & Frecuencia & Porcentaje & $\begin{array}{c}\text { Porcentaje } \\
\text { válido }\end{array}$ & $\begin{array}{c}\text { Porcentaje } \\
\text { acumulado }\end{array}$ \\
\hline Deficiente & 5 & 1,9 & 1,9 & 1,9 \\
Bajo & 49 & 18,8 & 18,8 & 20,8 \\
Regular & 98 & 37,7 & 37,7 & 58,5 \\
Bueno & 108 & 41,5 & 41,5 & 100,0 \\
Total & 260 & 100,0 & 100,0 & \\
\hline
\end{tabular}

En la Tabla 2, se observa que en el rendimiento académico de los estudiantes, es el $41,5 \%$ bueno; $37,7 \%$ regular y un $18,8 \%$ niveles bajo. 
Tabla 3. Correlación del clima social familiar con el rendimiento académico

\begin{tabular}{|c|c|c|c|c|}
\hline & & & $\begin{array}{c}\text { Clima Social } \\
\text { Familiar }\end{array}$ & Rendimiento Académico \\
\hline \multirow[t]{6}{*}{ Rho de Spearman } & $\begin{array}{l}\text { Clima Social } \\
\text { Familiar }\end{array}$ & $\begin{array}{l}\text { Coeficiente de } \\
\text { correlación }\end{array}$ & 1,000 & $484^{* *}$ \\
\hline & & Sig. (bilateral) & . & ,000 \\
\hline & & $\mathrm{N}$ & 260 & 260 \\
\hline & $\begin{array}{l}\text { Rendimiento } \\
\text { Académico }\end{array}$ & $\begin{array}{l}\text { Coeficiente de } \\
\text { correlación }\end{array}$ &, $484^{* *}$ & 1,000 \\
\hline & & Sig. (bilateral) & ,000 & . \\
\hline & & $\mathrm{N}$ & 260 & 260 \\
\hline
\end{tabular}

Por otro lado, el modelo estadístico Rho Spearman reporta un coeficiente de correlación de 0.48 con $p$ valor $=0.000$ dónde $p<\alpha(0.05)$, lo cual indica que la correlación es casi media , directa y significativa. Lo que confirma la correlación de las variables. Por lo cual se concluye que; si existe relación significativa entre el Clima social familiar y el rendimiento académico en la Institución Educativa José Félix Black de Paiján, La Libertad, 2015.

Tabla 4. Correlación de las relaciones familiares con el rendimiento académico

\begin{tabular}{|c|c|c|c|c|}
\hline & & & Relaciones & $\begin{array}{c}\text { Rendimiento } \\
\text { Académico }\end{array}$ \\
\hline \multirow[t]{6}{*}{$\begin{array}{l}\text { Rho de } \\
\text { Spearman }\end{array}$} & Relaciones & $\begin{array}{l}\text { Coeficiente de } \\
\text { correlación }\end{array}$ & 1,000 &, $474^{* \star}$ \\
\hline & & Sig. (bilateral) & · & ,000 \\
\hline & & $\mathrm{N}$ & 260 & 260 \\
\hline & $\begin{array}{l}\text { Rendimiento } \\
\text { Académico }\end{array}$ & $\begin{array}{l}\text { Coeficiente de } \\
\text { correlación }\end{array}$ &, $474^{\star *}$ & 1,000 \\
\hline & & Sig. (bilateral) & ,000 & . \\
\hline & & $\mathrm{N}$ & 260 & 260 \\
\hline
\end{tabular}

Por otro lado, el modelo estadístico Rho Spearman reporta un coeficiente de correlación de 0.47 con $p$ valor $=0.000$ dónde $p<\alpha(0.05)$ lo cual indica que la correlación es casi media, directa y significativa. Lo que confirma la correlación de las variables. Por lo cual se concluye que existe relación directa y significativa entre la dimensión relaciones familiares y el rendimiento académico de los estudiantes de educación secundaria en la Institución Educativa José Félix Black de Paiján, La Libertad, 2015. 
Tabla 5. Correlación del desarrollo familiar con el rendimiento académico

\begin{tabular}{|c|c|c|c|c|}
\hline & & & $\begin{array}{c}\text { Rendimiento } \\
\text { Académico }\end{array}$ & $\begin{array}{c}\text { Desarrollo } \\
\text { Familiar }\end{array}$ \\
\hline \multirow{7}{*}{$\begin{array}{l}\text { Rho de } \\
\text { Spearman }\end{array}$} & Rendimiento & Coeficiente de & \multirow{2}{*}{1,000} & \multirow{2}{*}{$464^{\star *}$} \\
\hline & Académico & correlación & & \\
\hline & & Sig. (bilateral) & . &, 000 \\
\hline & & $\mathrm{N}$ & 260 & 260 \\
\hline & \multirow[t]{3}{*}{ Desarrollo Familiar } & $\begin{array}{l}\text { Coeficiente de } \\
\text { correlación }\end{array}$ & $464^{* *}$ & 1,000 \\
\hline & & Sig. (bilateral) &, 000 & . \\
\hline & & $\mathrm{N}$ & 260 & 260 \\
\hline **. La corre & n es significativa en & nivel 0,01 ( 2 cola & & \\
\hline
\end{tabular}

Por otro lado, el modelo estadístico Rho Spearman reporta un coeficiente de correlación de 0.46 con $p$ valor $=0.000$ dónde $p<\alpha(0.05)$, lo cual indica que la correlación es casi media, directa y significativa. Lo que confirma la correlación de las variables. Por lo cual se concluye que; Existe relación directa y significativa entre el desarrollo familiar y el rendimiento académico de los estudiantes de educación secundaria en la Institución Educativa José Félix Black de Paiján, La Libertad, 2015.

Tabla 6. Correlación de la estabilidad con el rendimiento académico

\begin{tabular}{lllcc}
\hline & & $\begin{array}{c}\text { Rendimiento } \\
\text { Académico }\end{array}$ & Estabilidad \\
\hline Rho de & Rendimiento & Coeficiente de & 1,000 &, $363^{* *}$ \\
Spearman & Académico & correlación & &, 000 \\
& & Sig. (bilateral) & 260 & 260 \\
& $N$ & Coeficiente de &, $363^{* *}$ & 1,000 \\
& Estabilidad & correlación &, 000 &. \\
& & Sig. (bilateral) & 260 & 260 \\
& $N$ & & \\
\hline
\end{tabular}

Por otro lado, el modelo estadístico Rho Spearman reporta un coeficiente de correlación de 0.36 con $p$ valor $=0.000$ dónde $p<\alpha(0.05)$ lo cual indica que la correlación es casi media, directa y significativa. Lo que confirma la correlación de las variables. Por lo cual se concluye que; Existe relación directa y significativa entre la estabilidad familiar y el rendimiento académico de los estudiantes de educación secundaria en la Institución Educativa José Félix Black de Paiján, La Libertad, 2015. 
DISCUSION

Los datos descriptivos del rendimiento académico igualmente presenta promedio regular el $37,7 \%$ un grupo muy representantivo el $41,5 \%$ en niveles bueno de rendimiento y por otro lado un $18,8 \%$ en niveles bajo. Estos referencias estadísticas demuestran que mientras en el aspecto inferencial se demuestra una relación media y significativa en el aspecto descriptivo tiende a esparcirse los resultados un buen porcentaje de estudiantes en ambos extremos tanto en niveles alto y bajo. Dicho de otro modo, el Clima social familiar en los estudiantes si bien los datos evidencia cierto grado de implicancia (en forma casi media) en el rendimiento académico se presente un poco diferente; porque la variable rendimiento académico es una variable que puede ser determinada por otros factores.

Se logró así con este artículo mostrar varias relaciones significativas del rendimiento académico con el clima social familiar además de sus dimensiones como, por ejemplo la dimensión "relaciones familiares" y el rendimiento académico que se evidencia una relación directa casi media (0.47); y significativa (0.000). Esto quiere decir que las "relaciones familiares": que evalúa el grado de comunicación y libre expresión dentro de la familia de los alumnos y el grado de interacción conflictiva está implicando de forma casi media en el promedio académico de los alumnos de esta institución educativa. Como corrobora los resultados de los indicadores descriptivos que 51,9\% de estudiantes presentan "relaciones familiares" de forma regular. El $65 \%$ de los estudiantes de este colegio presentan una cohesión alta; es decir: el grado en el que los miembros del grupo familiar están compenetrados y se apoyan entre sí; el 43,8\% presentan expresividad en forma alta es decir que los miembros de la familia actúa libremente y expresan sus sentimientos.

Asimismo, la mitad del alumnado 49,3\% evidencia niveles de conflicto bajo; grado en el que expresan libre y abiertamente la cólera agresividad y conflicto entre los miembros de la familia de los alumnos de este colegio. El análisis de dichos resultados descriptivos consolidan el grado de implicancia de forma directa que tiene la dimensión "relaciones familiares" con el rendimiento académico en esta casa de estudios. Aspecto que coinciden con los resultados de Fuentes (2003, citado por Gonzales y Pereda, 2006) quien concluyó que el Clima afectivo familiar así como la falta de preocupación de los padres por los deberes escolares de sus hijos(as) influye en el rendimiento escolar. Los resultados permiten comprobar que en las familias de estos alumnos, se vivencia un clima negativo, por cuanto experimentan una carencia de afecto, cariño y ternura, padres centrados más en lo negativo y la crítica, familias que no ofrecen seguridad $y$ confianza a sus hijos.

Por otro lado, en cuanto a la relación del grado de implicancia que tiene el "desarrollo familiar" con el rendimiento académico con una relación directa casi media (0.45); y significativa (0.000). Dicho de otro modo, en cuanto al "desarrollo familiar": la evaluación de ciertos procesos de desarrollo personal entre ellos autonomía, actuación, intelectual-cultural y 
moralidad-religiosidad dentro de la familia de los alumnos están implicando de forma casi media en el promedio académico de los alumnos de esta institución educativa. Un aspecto que difiere con Eñoki (2006,citado por Gonzales, 2006) que también hizo un estudio en la zona de la Libertad en la provincia de Viru pero no hallo relación significativa en las sub. escalas expresividad, conflicto, autonomía, actuación, intelectualcultural, socio recreativa, moralizaciónreligiosidad, organización y rendimiento académico. Un aspecto que puede estar reflejando la influencia de la cultura en la forma de ser de los hogares.

Detallando, en el contexto de la dimensión "desarrollo familiar" ; corrobora los resultados de sus indicadores descriptivos que el $45 \%$ de estudiantes presentan un clima de "desarrollo familiar" de forma regular. El $45,4 \%$ de los estudiantes de este colegio presentan un clima social familiar con una autonomía en niveles regulares; es decir que los miembros de sus familias están seguros de sí mismos, son independientes y toman sus propias decisiones de forma regular. Asimismo, el $51,5 \%$ niveles de actuación regular direccionado hacia niveles bajo de actuación el $48,5 \%$ dicho de otro modo las actividades, tales como el colegio o el trabajo se enmarca en una estructura orientada a la acción en forma regular hacia abajo.

De la misma forma en el mismo contexto del "desarrollo familiar" en cuanto al indicador "intelectual cultural" es decir el grado de interés que tienen la familia de los alumnos en las actividades de tipo político, intelectual y cultural y social que impactan en sus hijos se evidencia también que un poco más de la mitad $52,3 \%$ de alumnos presentan también niveles regulares de ese indicador. Igualmente, el indicador "social recreativo" el 42,3\% de los alumnos de este colegio presentan niveles regulares de aspectos sociales recreativos y el mas del $50 \%$ en niveles altos de moralidad religiosa aspectos que implican en cierta medida en el rendimiento académico. Resultados que corroboran lo referido por Sánchez (2013) quien resalto que a mayor participación de los padres en la educación de sus hijos, mayor será el aprovechamiento de los mismos, dentro del avance que los alumnos pueden lograr cuando se habla de buenas calificaciones, además indicó que es importante nombrar el control de los padres hacia sus hijos, representado por el tiempo de ocio de los niños, cantidad y tipo de literatura a la que tiene acceso, tareas así como las facilidades que tiene para realizarla.

De igual modo, en referencia a la dimensión "estabilidad familiar" en cuanto al rendimiento académico se refleja una correlación moderada, directa en un grado 0,36\% y significativa 0,000 ; es decir: el $63,5 \%$ del alumnado encuestado presenta niveles altos de organización y estructura al planificar las actividades y responsabilidades en sus familias y los que en su vida familiar tienen control $47,7 \%$ en niveles altos se atiene a reglas $y$ procedimientos establecidos presentan también niveles considerables de rendimiento académico. Resultados que coinciden con lo que concluyo Eñoki (2006, citado por Gonzales, 2006) que existe una relación significativa 
entre la sub escala control y rendimiento académico. También coincide con lo que halló Zavala (2001) quien en sus conclusiones refiere que: el $53.5 \%$ de los alumnos evaluados en su estudio expresan que el clima familiar que vivencian presenta una estructura inadecuada, caracterizada por la inestabilidad, mientras que ningún alumno del grupo evaluado refiere vivenciar un ambiente estable en su hogar, el $47.6 \%$ comunicando que no se sienten apoyados por los miembros de su familia al momento de tomar decisiones o actuar, mientras que el $39.2 \%$ de alumnos evaluados manifiestan vivenciar inadecuados niveles de comunicación; aspectos que implican de alguna manera en su bajo rendimiento académico.

Finalmente, el presente estudio corrobora el resultado de otros estudios que refieren que el Clima Familiar se relaciona con el Rendimiento Académico (Velásquez 2014, Gonzales, 2006) como bien lo afirma Coello (2010) quien afirmo que el $66 \%$ de alumnos que provienen de hogares completos aprobaron el año sin complicaciones. Aunque la variable rendimiento académico es un constructo se sensible a otros factores cabe resaltar que el clima social familiar impacto en algún modo en que el alumno pueda tener mejor eficiencia en sus estudios, además de considerar que el desarrollo de un buen clima social familiar está supeditado a factores culturales de la zona, además de la educación, religión y otros que configuran un buen clima social familiar.

\section{CONCLUSIONES}

El objetivo de la presente investigación fue determinar la relación del Clima social familiar y el rendimiento académico de los estudiantes de educación secundaria en la Institución Educativa José Félix Black de Paiján, La Libertad, 2015. Hallándose que el Clima social familiar y el rendimiento académico están relacionados de forma casi media, positiva y significativa en los estudiantes de educación secundaria. Dicho de otro modo, en la presente Institución Educativa los estudiantes que desarrollan un buen clima la observación general que los estudiantes que mantienen buen clima familiar también tienen buen rendimiento académico caso contrario los que mantienen un clima social familiar nocivo también ponen en riesgo su rendimiento académico en la presente Institución. Un aspecto que es corroborado con los resultados descriptivos que muestran que la mayoría de estudiantes mantienen un Clima Familiar normal o con características de un hogar promedio (83\%) y son muy pocos que evidencian niveles altos de buen clima $(12,7 \%)$ y en niveles bajo sólo (4.2\%).

\section{REFERENCIAS}

Bronfenbrenner, U. (1976). The experimental ecology of education. Educational researcher, 5(9), 5-15

Cassullo, G. L., Álvarez, L. V., y Pasman, P. (1998). Adaptación de las escalas de clima social escolar y familiar, VI Anuario de Investigaciones 
Coello, M. (2010). Incidencia de la estructura y clima familiar en el rendimiento escolar de los alumnos del octavo, noveno y décimo año de educación básica de la unidad educativa salesiana "José Felix Pintado" durante el año lectivo 20082009. Tesis de licenciatura. Universidad Politécnica Salesiana

Eñoki, Y. (2006). Clima Social Familiar y Rendimiento Académico de los alumnos de $4^{\circ}$ y $5^{\circ}$ Grado de educación Secundaria de la Institución Educativa Privada Virgen de Guadalupe . Virú

Fernández, B. (1998). Protagonismos de los padres de familia. Perú: Editorial family.

Fuentes, I. (2003). Influencia del Clima Afectivo Familiar en el Rendimiento escolar de los alumnos del $3^{\circ}$ año $\mathrm{C}$ y $7^{\circ}$ año C de la Escuela Emilia Romagna de Traiguén. Chile

Gonzales P. y Pereda I. (2006). Relación entre el clima social familiar y el rendimiento escolar de los alumnos de la I.E San Santiago. Chimbote

Kemper, S. (2000). Influencia de la práctica religiosa y del género de la familia sobre el clima social familiar. Lima

López-Justicia, M.D. Fernández de Haro, E., Amezcua, J.A. y Pichardo, M. C. (2000). Difieren en autoconcepto los adolescentes con baja visión de los adolescentes con visión normal ? integración 33, 14-20
Ministerio de Educación. (2008). Diseño curricular nacional de la Educación Básica Regular. Pg. 53. Resolución Ministerial Nº440 - 2008 - ED. Pg. 53. URL Recuperado de http://es.slideshare.net/juanjose2010/dc n-2009-11909818

Mizala, A. y. (2002). Factores que inciden en el Rendimiento Escolar en Bolivia. Universidad de Chile

Moos, R. (1984). Escalas de clima social: Familia, trabajo, instituciones correccionales y aulas. Madrid: TEA

Murillo, F.J. (2008). Enfoque, situación y desafíos de la investigación sobre eficacia escolar en América Latina y el Caribe. En UNESCO, Eficacia escolar y factores asociados en América Latina y el Caribe. Santiago de Chile: UNESCO

Sánchez, I. (2013). Apoyo parental y rendimiento académico. México

Williams, N. (1993) Una aproximación a la medición del clima o ambiente de un sistema social-familiar, Comportamiento, Vol. 2, N¹, Universidad Simón Bolívar, Caracas, Venezuela

Zavala García, G. (2001). Clima familiar y su relación con los intereses vocacionales y los tipos caracterológicos de los alumnos del 5to año de secundaria de los colegios nacionales del distrito del Rímac. Lima 